\title{
Haematospermia - a systematic review
}

\author{
PRIYA KUMAR, SONA KAPOOR, VINOD NARGUND
}

\section{Department of Urology, St Bartholomew's and Homerton University Hospitals, London, UK}

\section{ABSTRACT}

Haematospermia (or haemospermia) is a distressing symptom in sexually active men. In most cases, it is caused by non-specific inflammation of the prostate and seminal vesicles. In a small percentage of men, however, it may be a manifestation of genitourinary or systemic malignancy, in particular prostate cancer. The purpose of this review is to explain the causes and management of patients with haematospermia.

\section{KEYWORDS}

Haematospermia - Review

\section{CORRESPONDENCE TO}

Mr VH Nargund, Consultant Urological Surgeon, St Bartholomew's Hospital, West Smithfield, London EC1A 7BE, UK

T: +44 (0)20 7601 8394; F: +44 (0)20 7601 7844; E: Vinodnargund@yahoo.co.uk

Haematospermia is defined as the appearance of blood in the ejaculate. It has been documented by physicians for centuries from Hippocrates, Galen, Paré, Morgagni to Fournier. ${ }^{1}$ Although it is a painless, benign and self-limiting symptom in most cases, its appearance may be frightening and alarming to the patient. In the past, urologists have generally regarded haematospermia as a benign condition and, as such, recommended conservative therapy. More recently, however, an association between prostate cancer and haematospermia has been established. ${ }^{2,3}$ In a recent study looking at the incidence of prostate cancer in patients with haematospermia, Han et al. ${ }^{3}$ reported the diagnosis of prostate cancer in nearly $14 \%$ of men presenting with haematospermia.

For haematospermia to occur, intact emission and ejaculation functions are necessary. ${ }^{4}$ The exact incidence of haematospermia remains unknown as most ejaculates go unnoticed during intercourse. It may be indicative of a significant underlying genito-urinary condition particularly in older patients where it may be a symptom of prostatic neoplasia. A methodical approach to its management, therefore, should be employed. ${ }^{5}$

Haematospermia should be investigated by a thorough clinical assessment and non-invasive investigations. The main aim is to exclude any underlying pathology and, if none is found, then to re-assure the patient after full evaluation.

\section{Aetiology}

With increasing information in the literature and advances in radiological imaging, the causes of haematospermia may be subclasssified according to different aetiological origins congenital, inflammatory, infective and systemic (Table 1).

\section{Clinical assessment}

Most patients who notice haematospermia consult their doctor promptly after the first episode. Like other obscure clinical conditions, systematic clinical history and assessment help to evaluate the causation of haematospermia. It is also necessary to know whether haematospermia is an isolated or a recurrent episode.

There are two main aims in the evaluation: first, to ensure that there is no underlying sinister cause or a specific condition that is treatable thereby alleviating the patient's symptoms; second, to re-assure the patient if no causative factor is found. It is important, however, to make sure that the patient is actually describing haematospermia and not haematuria; in some cases, both may co-exist. If haematuria is present with haematospermia, the haematuria is investigated according to the standard protocols.

Most men with haematospermia are likely to be less than 40 years old with symptoms ranging from a few weeks to a few months in duration. The likelihood of recurrent haematospermia is seen in the older age group.

Sexually transmitted diseases may point to an infective cause such as urethritis, prostatitis, epididymitis, HIV or condylomata. Other infective causes include bilharziasis, ${ }^{6}$ cytomegalovirus $^{7}$ and hydatid disease. ${ }^{8}$ Associated lower urinary tract symptoms should raise the possibility of a urethral stricture or a vascular and enlarged prostate. The patient is asked as to whether there was any trauma or selfinstrumentation in the urogenital region. A history of prolonged and intense masturbation or sexual intercourse could lead to the congestion of genital organs and bleeding.

Transrectal ultrasound biopsy of the prostate is invariably associated with haematospermia. Systemic review 


\section{Table 1 Causes of haematospermia}

$\begin{array}{ll}\text { Congenital } & \text { Seminal vesicle (SV)/ejaculatory duct cysts } \\ \text { Inflammatory } & \text { Urethritis, prostatitis, epididymitis } \\ & \text { Genito-urinary tuberculosis } \\ & \text { Cytomegalovirus, HIV } \\ & \text { Schistosomiasis, hydatid } \\ & \text { Condylomata of urethra and meatus } \\ & \text { Urinary tract infection } \\ \text { Obstruction } & \text { Prostatic and SV and } \\ & \text { ejaculatory duct calculi } \\ & \text { Postinflammatory SV cysts } \\ & \text { SV diverticula } \\ & \text { Urethral stricture } \\ \text { Utrical cyst } & \text { Benign prostatic hypertrophy } \\ \text { Tumours } & \text { Prostate* } \\ & \text { Bladder* } \\ \text { SV } \\ \text { Urethra } \\ \text { Testis*, epididymis } \\ \text { Melanoma } \\ \text { Prostatic varices } \\ \text { Prostatic telengectasia } \\ \text { Haemangioma } \\ \text { Posterior urethral veins } \\ \text { Excessive sex, masturbation }\end{array}$

Trauma/

iatrogenic

Perineum
Testicle
Self-instrumentation
Post-haemorrhoid injection, TRUS biopsy
Vaso-venous fistula

Systemic Hypertension

Haemophilia

Purpura

Scurvy

Bleeding disorders

Chronic liver disease

Renovascular disease

Leukaemia*

Lymphoma*

Cirrhosis of the liver

Amyloidosis

Idiopathic

*Important conditions to be ruled out in patients with hematospermia. should include any history of hypertension, ${ }^{9}$ renal, liver or bleeding disorders. ${ }^{5}$

Physical examination may help to locate the source of haematospermia. A full general examination including an assessment of blood pressure should be performed. The abdomen is examined for hepatosplenomegaly, masses and palpable bladder. The testes, epididymis, cord and penile urethra are carefully examined for inflammatory and neoplastic pathology. The urethral meatus needs to be examined for the evidence of trauma, condylomata, phimosis and cancer. Testicular tumours may occasionally present with haematospermia. ${ }^{10}$ Testicular and epididymal examination will also reveal the presence of infective element including tuberculous disease. Palpation of the vasa deferentia may show thickening, induration or nodularity due to tuberculosis. Rectal examination will allow assessment of the prostate gland. Tenderness or induration of the prostate may imply infection whilst a hard texture or nodule may be indicative of neoplasia especially in an older individual.

If the seminal vesicles are palpable then there could be associated pathology and an underlying abnormality must be excluded with further imaging investigations.

\section{Investigations}

If the history needs confirmation, a 'condom test' can be carried out where the patient is asked to collect the ejaculate in the condom which is examined for blood. ${ }^{4}$ Urinalysis and urine culture will help to confirm the presence of urinary infection and haematuria, which if present will require further radiological investigations where indicated. Urine cytology although not mentioned in the literature is a simple test to rule out the bladder pathology.

If a sterile pyuria is noted then further investigations are required to exclude tuberculosis as an underlying pathology. Semen analysis may show the presence of pus cells which then warrants further investigation in search of an infectious aetiology; this includes semen culture, urethral swabs, mycobacterial cultures and viral serology.

Serum coagulation profile may reveal underlying bleeding disorders whilst the erythrocyte sedimentation rate (ESR) may be raised in tuberculosis. If sexually transmitted diseases are suspected, urethral cultures for gonorrhoea and chlamydia are obtained. In patients who have a suspicious nodule on rectal examination or who are in their late 40s (younger in patients with family history of prostate cancer), estimation of prostate specific antigen (PSA) is required.

\section{Imaging}

Further investigations depend on other symptoms and the clinical judgement of the clinician assessing the patient. Upper tract imaging is rarely necessary. 


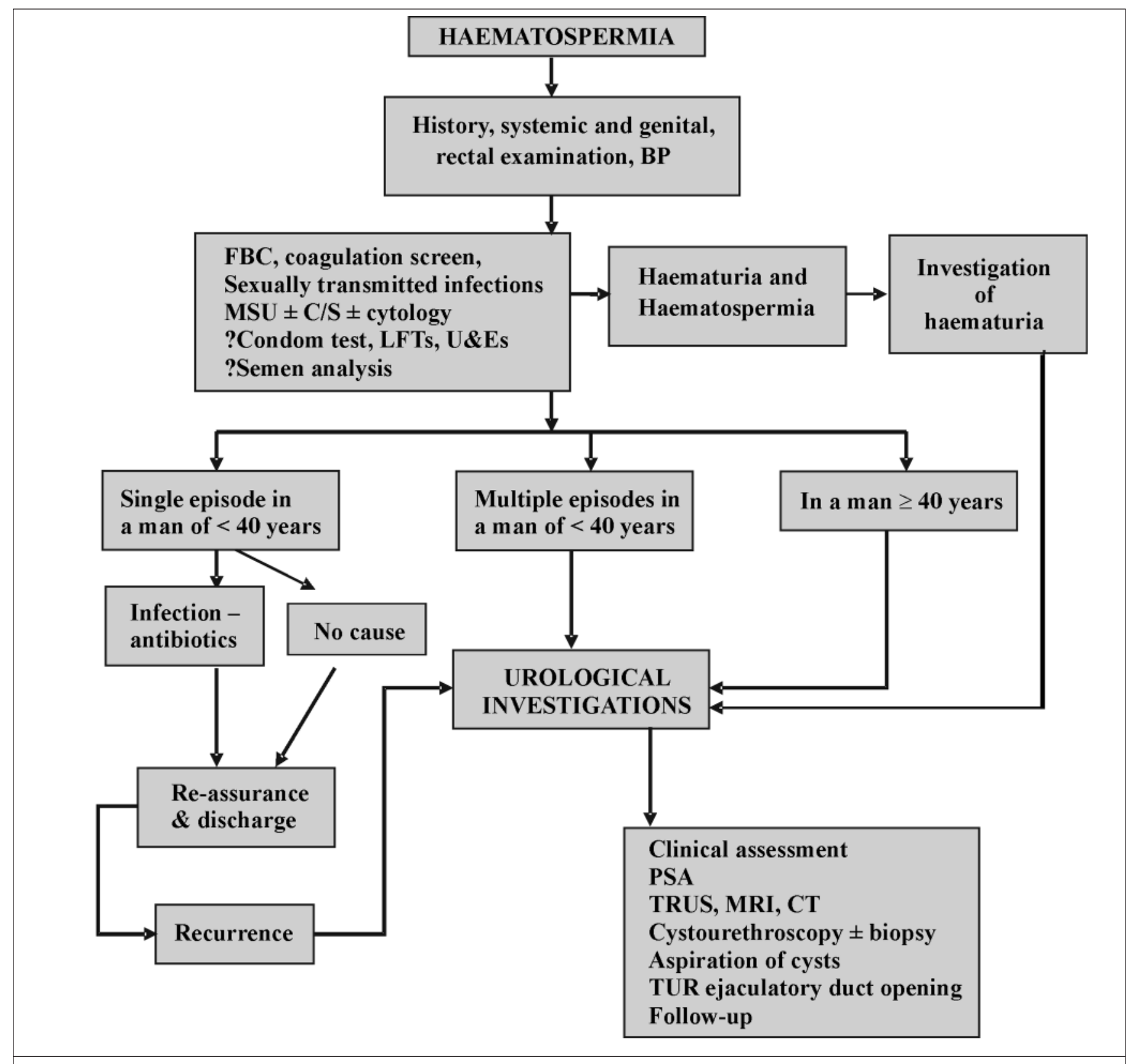

Figure 1 Algorithm for evaluation and management.

Transrectal ultrasonography (TRUS) may help in the diagnosis of prostatic and seminal vesicular pathology. The TRUS examination allows excellent visualisation of the prostate, seminal vesicles and adjacent structures. Various studies have shown its benefit in leading to a diagnosis namely the findings of calculi, cysts, mullerian duct remnants, prostatic varices and inflammatory changes. ${ }^{11,12}$ TRUS-guided cyst aspiration has also been described. If ultrasonography is entirely normal and the symptoms are persistent, it may be advisable to proceed with other imag- ing modalities like magnetic resonance imaging (MRI) both conventional and endorectal coil - and computerised tomography (CT) to improve visualisation of the anatomy of the pelvic organs. ${ }^{13}$ Any suspicious testicular swelling is investigated with an urgent ultrasound examination.

\section{Treatment}

Although imaging may reveal the underlying cause of haematospermia there is often little that can be offered in 
the form of conventional treatment. The principal aim of the management is to exclude serious conditions like prostate and bladder cancer and treat any other underlying cause. If no pathology is found, then it is important to allay the anxiety and re-assure the patient. The general concept of management is set out in Figure 1.

It is necessary to stress to the patient that haematospermia is rarely associated with a significant pathology. In a younger patient less than 40 years of age with a single episode of haematospermia, clinical assessment with basic investigations to rule out any inflammation and infection in the genito-urinary system is sufficient.

Individuals with recurrent haematospermia and who are middle-aged need specialist urological assessment and investigations. If infection is suspected, a course of 5aminoquinolones such as ciproxin or trimethoprim-sulphamethoxazole and doxycycline combination would be beneficial even if the urine cultures are negative. ${ }^{14}$ Systemic conditions are treated appropriately. Prostatic or seminal vesicular cysts can be aspirated under TRUS guidance. In recurrent haematospermia, cystourethroscopy may help to diagnose congested veins in the prostate and urethral abnormalities. Ejaculatory duct obstruction is managed by a transurethral incision at the duct opening. ${ }^{15}$

Persistent haematospermia presents difficulty from the management point of view and a detailed radiological assessment may ascertain the exact cause. It is also important to keep these patients under follow-up for a limited period. In some patients, persistent or recurrent haematospermia could be the only symptom of prostate cancer. In high-risk individuals - middle-aged or with family history of prostate cancer - surveillance with PSA over a period of time is desirable.

\section{References}

1. Mulhall JP, Albertson PC. Haemospermia: diagnosis and management. Urology 1995; 46: 463-7.

2. Rubinowicz DM, Soloway MS, Lief M, Civantos F. Hemospermia and expressed tumor in the urethra: an unusual presentation of ductal carcinoma of the prostate. J Urol 2000; 163: 915.

3. Han M, Brannigan RE, Antenor J-AV, Roehl KA, Catalona WJ. Association of hemospermia with prostate cancer. J Urol 2004; 172: 2189-92.

4. Munkelwitz R, Krasnokutsky S, Lie J, Shah SM, Bayshtok J, Khan SA. Current perspectives on hematospermia: a review. J Androl 1997; 18: 6-14.

5. Narouz N, Wallace DM. Haematospermia: in the context of a genitourinary medicine setting. Int J STD AIDS 2002; 13: 517-21.

6. Elem B, Patil PS. Haemospermia: observations in an area of endemic bilharziasis. Br J Urol 1987; 60: 170-3.

7. Koment RW, Poor PM. Infection by cytomegalovirus associated with chronic haemospermia. Urology 1983; 22: 617-21.

8. Whyman MR, Morris DL. Retrovesical hydatid causing haemospermia. Br J Urol 1991; 68: 100-1.

9. Bhaduri S, Riley VC. Haematospermia associated with malignant hypertension. Sex Transm Infect 1999; 75: 200.

10. Mahesh K, Gordon S, Otite U, Berney D, Nargund VH. Testicular tumour presenting as haematospermia. J Urol 2001; 165: 188.

11. Yagci C, Kupeli S, Tok C, Fitoz S, Baltaci S, Gogus O. Efficacy of transrectal ultrasonography in the evaluation of hematospermia . Clin Imaging 2004; 28: 286-90.

12. Worischeck JH, Parra RO. Chronic haemospermia: assessment by transrectal ultrasound. Urology 1994; 43: 515-20.

13. Maeda H, Toyooka N, Kinukawa T, Hattori R, Furukawa T. Magnetic resonance images of haematospermia. Urology 1993; 41: 499-504.

14. Ganabathi K, Chadwick D, Fenely RC, Gingell JC. Haemospermia. Br J Urol 1992; 69: 225-30.

15. Fuse $\mathrm{H}$, Nishio R, Murakami K, Okumura A. Transurethral incision for hematospermia caused by ejaculatory duct obstruction. Arch Androl 2003; 49: 433-8. 